\title{
A framework for robustness assessment in the context of corroded RC structures
}

\author{
Eduardo S. Cavaco \\ Universidade Nova de Lisboa, Civil Engineering Department, Monte de Caparica, Portugal
}

Joan R. Casas

Technical University of Catalunya, Civil Engineering Department, Barcelona Spain

Luis A. C. Neves

Universidade Nova de Lisboa, Civil Engineering Department, Monte de Caparica, Portugal

\author{
Alfredo H. Huespe \\ International Center for Computational Methods in Engineering (CIMEC), Santa Fe, Argentina
}

\begin{abstract}
Structural robustness is an emergent concept related to the structural response to damage. At the present time, robustness is not well defined and much controversy still remains around this subject. Even if robustness has seen growing interest as a consequence of catastrophic consequences due to extreme events, the fact is that the concept can also be very useful when considered on more probable exposure scenarios such as deterioration, among others. This paper intends to be a contribution to the definition of structural robustness, especially in the analysis of reinforced concrete structures subjected to corrosion. To achieve this, first of all, several proposed robustness definitions and indicators and misunderstood concepts will be analyzed and compared. From this point and regarding a concept that could be applied to most type of structures and damage scenarios, a robustness definition is proposed. To illustrate the proposed concept, an example of corroded reinforced concrete structures will be analyzed using nonlinear analysis numerical methods based on a continuum strong discontinuities approach and isotropic damage models for concrete. Finally the robustness of the presented example will be assessed.
\end{abstract}

\section{INTRODUCTION}

Tragic events such as the partial collapse of Ronan Point building (London, UK - 1968) or most recently the collapse of the World Trade Center (New York, USA - 2001) and the I-35W Mississippi River bridge (Minneapolis, USA - 2007), among others, increased the attention of engineers, but also the society, to the safety, reliability and robustness of structures.

Ronan Point was a 23-storey tower block in Newham, East London, which suffered a fatal partial collapse due to a natural gas explosion on a kitchen located on a corner flat on the 18th floor of the building.

The collapse of World Trade Center Towers on September 11 was triggered by an impact of two commercial aircrafts that damaged partially the perimeter steel tube design and leading to overall collapse.

The initiating event in the collapse of the I-35W bridge was the lateral shifting instability of the upper end of a diagonal member and the subsequent failure of a gusset plate on the center portion of the deck truss. Since the deck truss portion of the I-35W bridge was non-load-path redundant, the total col- lapse of the deck truss was likely once the gusset plates failed (NTSB 2008).

What seems to be common to these cases is the occurrence of disproportionate consequences to the initial cause or damage. If on the first two cases the collapse was triggered by extreme and low probability events, on the last case, failure occurred when structure was submitted to regular load conditions. As a result, it is understandable that robustness is also a useful concept even when applied on the context of more probable events such as design loads, deterioration scenarios, as considered on this paper, among others.

Although robustness seems definitely a desirable property, the fact is that consensus about a precise definition does not exist and a framework to assess it is still under development.

Most modern structural design codes provide detailed direction for verifying if a design is acceptable with regard to individual failure modes, which, in most cases, relates to damage of individual structural components. Unfortunately the codes are far less specific regarding requirements for reliability against system failure (Baker et al. 2008).

This limitation is more important as most structural failures are due to unexpected loads, design errors, errors during execution, unforeseen deterioration and poor maintenance which can not be 
prevented using conventional component based code checking formats (Canisius et al. 2007).

Regarding this scenario, the question arises of what can be done to improve this situation, since actual design codes contain no prescriptions to check against system failures and, consequently, the main causes of structural collapse can not be predicted and avoided.

There are no simple answers for this question, but certainly the risk could be minimized if structures would be designed to be less vulnerable to local damage no matter what causes it, or in other words if structures would be more robust. To achieve this it is important to have firstly a precise definition of robustness and that to propose measures to assess it.

\section{BACKGROUND ON ROBUSTNESS}

\subsection{Definition}

As previously stated, a precise definition for robustness still does not exist. Several works (Callaway et al. 2000; Agarwal et al. 2006; Wisniewski et al. 2006; Starossek 2008; Baker et al. 2008) made interesting attempts to define it.

From the proposed definitions there are some common key concepts that must be highlighted. These fundamental concepts can be summarized as event, damage, structural performance and consequences. A straight relationship exists between them, i.e., a certain structure exposed to an event may suffer from damage that leads to some degree of loss of structural performance. From this, direct and indirect consequences may arise depending on the type and degree of structural performance loss.

From the referred works, and having in mind the presented fundamental concepts several times used to define robustness it is understandable that some authors (Wisniewski et al. 2006; Starossek 2008) consider that a structure is robust if the relationship between the initial damage and the degree of structural performance is acceptable. On the other hand, remaining referred authors define an unrobust structure when there is a disproportionate relationship between the trigger event and the consequences resulting from it.

On the first case, robustness is seen as a structural property because only depends on the structure itself, i.e., irrespective of the events responsible for damaging the structure or the consequences resulting from it. On the second case, a wide concept is considered, because it is important to have in mind the structural context. Robustness is defined as property of both structure and environment because exposure and consequences depend on it.

At this stage, it is important to note that robustness, defined as a property of both structure and environment, is a much wider concept involving com- plex socio and economical variables, among others, escaping from the civil engineering domain. Robustness becomes more difficult to assess because it depends on the developments on structural environment. Imagine for instance a viaduct over a highway. Viaduct robustness will decrease with highway traffic increase because exposure to accidents and failure consequences will be higher. On the other hand, if robustness is defined as property of the structure it only depends on the degree of structural performance after damage occurs no matters the extent of consequences and what causes damages. In this case, robustness is a more confined concept, more easily accessible to civil engineers that can be defined by the follow sentence: robustness is a property of the structure itself and measures the degree of structural performance loss after damage occurrence.

This relation can have many forms, from service limit states to ultimate limit states. Damage can vary from a simple degradation state to a more serious damage, as a column or a beam failure, among others. The concept behind this definition is not limit neither the functions spectrum nor the damage scenarios.

\subsection{Assessment}

The ambiguity on robustness subject is due, not only to the two distinct approaches about the definition, but also because there is a tendency to compare the proposed frameworks to measure it, or other related concepts, when, in fact, they measure different things as will be demonstrated.

On the next paragraphs the proposed measures for robustness and related concepts will be presented on a historical perspective.

Frangopol and Curley (1987) proposed a probabilistic redundancy factor, $\beta_{R}$, to account for the random nature in safety evaluation of damaged structures:

$\beta_{R}=\frac{\beta_{\text {Intact }}}{\beta_{\text {Intact }}-\beta_{\text {damaged }}}$

where $\beta_{\text {Intact }}$ is the reliability index of the intact system and $\beta_{\text {damaged }}$ is the reliability index of the damaged system. $\beta_{R}$ is equal to 1 when the damaged structure has no reserve strength and is infinite when the damage has no influence on the reserve strength of the bridge.

Lind (1995) proposes quantitative measures for the vulnerability and damage tolerance of a system. Vulnerability and damage tolerance are considered complementary concepts. The vulnerability, $V$, of a system is defined as:

$V=\frac{P\left(r_{0}, S\right)}{P\left(r_{d}, S\right)}$ 
where $r_{d}$ is the resistance of the damaged system, $r_{0}$ is the resistance of the intact system, and $S$ is the loading. $P(r, S)$ is the probability of system failure as a function of both effect of loading and resistance. The vulnerability $V$ of a system can vary from zero to infinite, if the damage has null or huge impact on system resistance, respectively. The system damage tolerance $T_{d}$ can be obtained inverting equation (2).

Ghosn and Moses (1998) proposed an entire methodology to assess system safety of highway bridges sub and superstructures. The incorporation of system behavior to the safety assessment is done by the relative reliability index $\Delta \beta$, which is defined as the difference between the safety index for the system and the safety index for the member. In order to guarantee the bridge safety, the obtained relative reliability index must be greater than the corresponding target values and, at the same time, the member safety has to be ensured. Considering for instance a damaged superstructure, the safety assessment of the bridge is satisfied when the following condition is verified:

$$
\Delta \beta_{\text {damaged }}=\beta_{\text {damaged }}-\beta_{\text {member }} \geq-2.70
$$

where $\Delta \beta_{\text {damaged }}$ is the relative reliability index of the damaged bridge and $\beta_{\text {damaged }}$ and $\beta_{\text {member }}$ are the reliability index of the damaged bridge and member respectively. The target value of $\Delta \beta_{\text {damaged }}$ was proposed by Ghosn and Moses (1998) based on the values found on bridges considered to having a robust behavior.

Baker et al. (2008) proposed a risk-based framework for robustness. Robustness is assessed by computing the ratio between direct and total risk. Direct $\left(R_{D i r}\right)$ and indirect risk $\left(R_{I n d}\right)$, which sum gives the total risk, are associated respectively with the direct and indirect consequences of the damaged system. Indirect consequences can be interpreted as the penalties disproportionate to the cause of damage, and so robustness of a system is indicated by the contribution of the indirect risks to total risk. The robustness index $I_{R o b}$ is then defined by equation (4):

$$
I_{R o b}=\frac{R_{D i r}}{R_{D i r}+R_{\text {Ind }}}
$$

The index may assume values between zero and one. If the system is completely robust, $I_{R o b}$ is equal to one, if all risk is due to indirect consequences, then $I_{R o b}$ is equal to zero.

As previously stated, robustness concept can also be useful on the context of ordinary events such as deterioration scenarios. Biondini and Restelli (2008) consider a trussed system subjected to corrosion of each of its members considered equivalent to the reduction on the effective cross section area. In order to assess robustness, they measure the ratio between several structural performance indicators in the in- tact state and in the deteriorated state. For corrosion levels from $0 \%$ to $100 \%$, robustness index $\rho$, is then given by:

$\rho=\frac{f_{0}}{f_{d}}$

where $f_{0}$ and $f_{d}$ are the structural performance indicators on the intact and deteriorated state respectively. Considering damage as a continuous variable, can be essential, since a structure may be robust for a certain damage level and suddenly became unrobust for a damage level slightly superior. The negative aspect of this approach is that robustness has different values for the same type of damage, which might contribute to bring more ambiguity to the subject.

Starossek (2009) proposed several robustness measures depending on the type of progressive collapse occurred. Among the proposed measures, the Damaged Based Robustness Measure II, obtained through equation (6), mainly measures the proportion between the direct consequences, $d$, and the initial damage $i$.

$$
R_{d, \text { int }}=1-2 \int_{0}^{1}[d(i)-i] d i
$$

$R_{d, \text { int }}$ is the integral damage based measure of robustness, varying from 0 to 1 when a structure has null or full robustness respectively.

This approach has the same advantage of the previous one because consider damage as a continuous variable. On the other hand, in this case, there is a single value for each damage type.

\subsection{Robustness assessment framework}

The lack of consensus about robustness is, in part, due to the direct comparison that has been done between the measures presented in the previous section. In fact, they can not be directly compared because they measure different things. On the other hand, some proposed measures are coherent with the structural property definition for robustness, and others are consistent with the notion of robustness being a property of both structure and its context. Equation (7), several times used to describe collapse, can be useful to understand how the proposed measures are related.

$$
P(F)=P(E) \times P(D \mid E) \times P(F \mid D)
$$

In equation $(7), P(E)$ is the probability of a certain exposure, $P(D \mid E)$ is the probability of the structure being damaged given an exposure and finally $P(F \mid D)$ is the failure probability of the damaged structure. The first term of equation (7) depends on structural context and the term $P(D \mid E)$ measures the vulnerability of the structure to the given exposure. Finally the term $P(F \mid D)$ measures the internal structural resistance to damage, and for that, it is a property of the structure closely related with the ap- 
proach that defines robustness as structural property. According with this judgment equation (7) could be rewritten in the following qualitative form:

\section{Failure $=$ Exposure $\times$ Vulnerability $\times$ Robustness}

In the next paragraphs, the presented probabilistic measures for robustness will be rewritten, considering a certain exposure $E$ and a specific damage scenario $D$ :

- Frangopol and Curley (1987) redundancy index:

$$
\beta_{R}=\frac{\phi(1-P(F))^{-1}}{\phi(1-P(F))^{-1}-\phi(1-P(F \mid D))^{-1}}
$$

- Lind (1995) damage tolerance index:

$$
T_{d}=\frac{1-P(F \mid D)}{1-P(F)}
$$

- Ghosn and Moses (1998) target value for the damaged system:

$$
\Delta \beta_{\text {damaged }}=\phi(1-P(F \mid D))^{-1}-\phi(1-P(D \mid E) P(E))^{-1}
$$

- Baker et al. (2008) risk based robustness measure:

$$
I_{R o b}=\frac{P(\bar{F} \mid D) C_{d i r}}{P(\bar{F} \mid D) C_{d i r}+P(F \mid D) C_{\text {ind }}}
$$

At this stage it is possible to extract the following conclusions: i) Indices proposed by Frangopol and Curley (1987) and Lind (1995) are very similar and mainly measure the importance of the term $\mathrm{P}(\mathrm{F} \mid \mathrm{D})$ to failure; ii) Ghosn and Moses (1998) proposal measure the difference between damaged system and member reliabilities; iii) Baker et al. (2008) proposal is conceptually different because it is a risk measure and for a damaged system it relates the consequences resulting from having a failure or not.

Although the robustness measure proposed by Biodini and Restelli (2008) is not probabilistic, it is closely related with the term $\mathrm{P}(\mathrm{F} \mid \mathrm{D})$ because it is a measure of the structural performance of the damaged system. The same can be said about Damaged Based Robustness Measure II proposed by Starossek (2009) with the difference that consequences of damaged system are evaluated instead of structural performance.

From the exposed it is understandable that the proposed robustness measures are closely related but, in rigor, they measure different things and for that it is abusive to compare directly themselves. There is no doubt that risk assessment is the target when discussing the viability of a project. So it might be acceptable that robustness index proposed by Baker et al. (2008) is the most correct measure.
However in order to assess this risk, it is necessary to evaluate all the terms in equation (7) and the associated consequences. Actual design codes give acceptable prescriptions to evaluate the terms $P(E)$ and $P(D \mid E)$, at least for regular exposures. On the other hand, a lack of knowledge still exists when it is necessary to compute the term $P(F \mid D)$ in order to assess system safety. For the term $P(F \mid D)$ evaluation, it is firstly necessary to compute system structural performance for the damage $D$ spectrum. A deterministic measure for robustness could be that obtained through equation (13), which gives the area above the curve defined by the normalized structural performance $f$ subjected to a normalized damage $d$ (See Figure 1).

$$
R_{d}=\int_{d=0}^{d=1} f(x) d x
$$

where $f$ is given by the ratio between the structural performances on the intact and damage states, and $d$ is given by the ratio between actual and maximum possible damage.

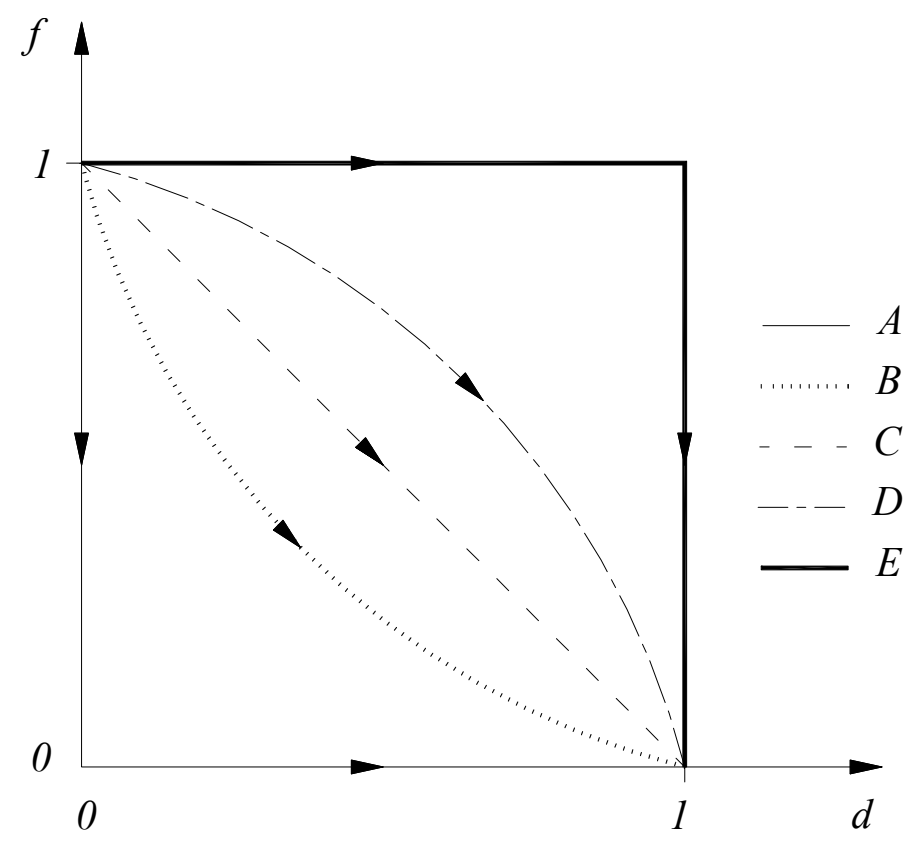

Figure 1. Robustness assessment. Normalized structural performance $f$ as a function of the normalized damage $d$.

Robustness index $R_{d}$ may vary from 0 to 1 respectively if a minimum damage level produces the entire loss of structural performance, curve $A$, or if the damage does not produces influence on structural performance, curve $E$. In Figure 1, curve $B, C$ and $D$ represent, respectively, intermediate increasing robustness.

This methodology is also valid in situations where the maximum damage does not correspond to total loss of the structural performance or in situations where the collapse happens before maximum damage occurs. 


\section{ROBUSTNESS OF A CORRODED RC BEAM}

\subsection{Introduction}

In order to illustrate the application of the previous proposed measure of robustness (see equation (13)), a simply supported footbridge (see Figure 2) subjected to corrosion of the longitudinal reinforcement was analyzed (Cavaco, 2009).

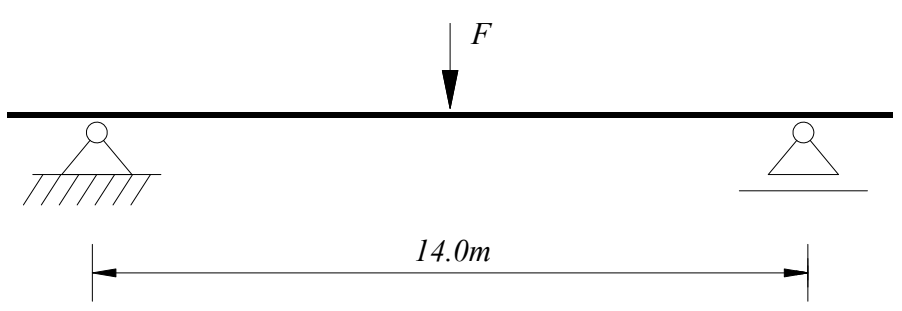

Figure 2. Simply supported footbridge under corrosion.

A simple cross section (see Figure 3) was designed in accordance with Eurocode prescriptions. Transversal reinforcement was overdesigned and considered protected against corrosion in order to simplify the analysis.

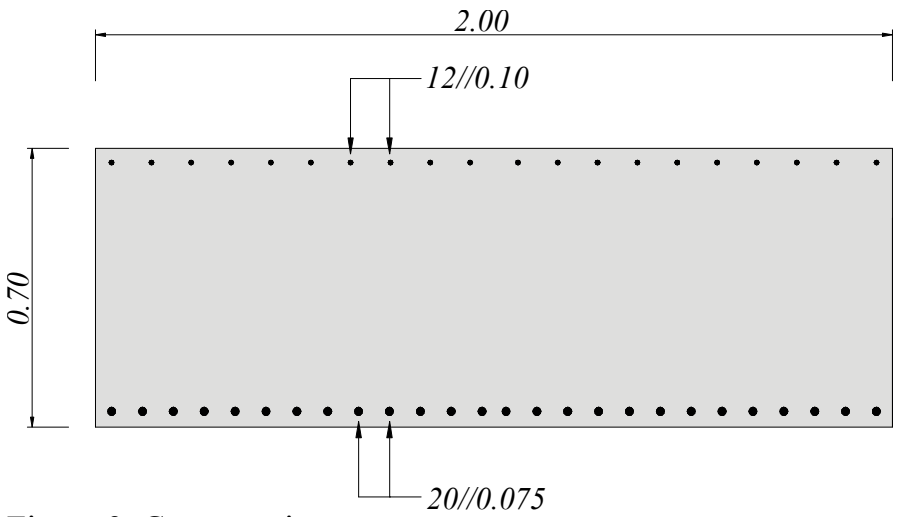

Figure 3. Cross section.

For concrete, material properties are presented in Table 1.

Table 1. Concrete Material Properties

\begin{tabular}{|c|c|c|c|c|c|}
\hline Material & $\begin{array}{l}\mathrm{f}_{\mathrm{ctm}} \\
\mathrm{MPa}\end{array}$ & $\begin{array}{l}\mathrm{f}_{\mathrm{cm}} \\
\mathrm{MPa}\end{array}$ & $\begin{array}{l}\mathrm{E} \\
\mathrm{GPa}\end{array}$ & $v$ & $\begin{array}{l}\mathrm{G}_{\mathrm{f}} \\
\mathrm{kN} / \mathrm{m}\end{array}$ \\
\hline Concrete & 3.0 & 30.0 & 30 & 0.20 & 0.1 \\
\hline
\end{tabular}

In Table $1, \mathrm{f}_{\mathrm{ctm}}$ and $\mathrm{f}_{\mathrm{cm}}$ are the maximum tension and compressive stresses. $\mathrm{E}, \mathrm{v}$ and $\mathrm{G}_{\mathrm{f}}$ are, respectively, the Young modulus, the Poisson coefficient and the fracture energy.

For steel reinforcement an elastoplastic constitutive behavior was considered with a yielding stress of $400 \mathrm{MPa}$ and a Young modulus of $200 \mathrm{GPa}$.

In order to analyze de effects of corrosion on RC structures a methodology proposed by Sánchez et al. (2008) was adopted. This methodology consists on two step analysis and its competence was demonstrated comparing numerical with experimental results. On the first step a cross section analysis is car- ried out with the objective of studying the effects of corrosion products expansion on section integrity. The results obtained are then used to build a 2D longitudinal model of the corroded structure in order to study its load carrying capacity and robustness.

Having in mind the robustness proposed definition and measure, damage considered is the corrosion level $X_{p}$ (in terms of weight) of the longitudinal reinforcement and the structural performance studied is the load carrying capacity $F$.

\subsection{Cross section analysis}

The first step of the referred methodology is to perform a cross section analysis with the objective of capturing the corrosion effects: expansion of corrosion products; concrete deterioration and concrete cracking and spalling. To achieve this, corrosion was modeled as a steel bar expansion and concrete was modeled using an isotropic continuum damage model, ICDM, (Oliver et al. 1990) coupled with the kinematics provided by the strong discontinuities approach, CSDA (Oliver et al. 2002). One of the main features of the ICDM is the consideration that, physically, the degradation of concrete strength is the result of the initiation, growth and coalescence of micro cracks and that this process may be modeled by introducing an internal damage variable, $d$, which can be a scalar quantity. When this constitutive model is coupled with the CSDA kinematics, it is possible to model concrete cracks, which are, from a macroscopical point of view, discontinuities that can be characterized as jumps on the displacement field across material.

A full detailed description of ICDM and CSDA can be found, respectively, in Oliver et al. (1990) and Oliver et al. (2002).

Corrosion products expansion was simulated by a volumetric deformation $\varepsilon^{0}$ of the steel bars. Assuming a plain strain state, the total strains $\varepsilon$ can be obtained by the sum of the strain due to stresses $\varepsilon^{e}$ and due to the referred volumetric expansion $\varepsilon^{0}$ :

$\varepsilon=\varepsilon^{e}+\varepsilon^{0}$

The volumetric expansion $\varepsilon^{0}$ can be obtained through the dilatational parameter $D$ applied incrementally during the time steps required to perform the non-linear analysis:

$D=\frac{R_{f}^{2}-R_{i}^{2}}{2 R_{i}^{2}}$

where $R_{i}$ and $R_{f}$ are respectively the initial and final bar radius.

With respect to the bond between reinforcement and concrete, an interface model proposed by Oliver et al. (2007) was adopted. This type of model together with the CSDA kinematics allows the expected separation between both materials when high corrosion levels are reached. 
Cross section analysis was performed for corrosion levels from $0 \%$ to $100 \%$. With corrosion increase, concrete around steel bars become damaged. In Figure 4, it is possible to observe damage in concrete due to steel bar expansion. Where damage variable $d$ is higher, displacements tend to concentrate. When damage reach the value of 1 , material loses all its strength and a jump on the displacement field appears, i.e. a crack, may occur.

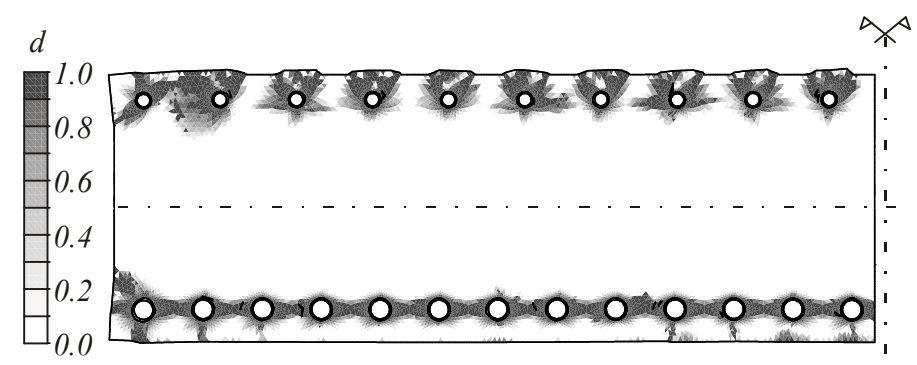

Figure 4. Damage $d$ in concrete due to steel bar expansion.

In Figure 5, isodisplacement lines are presented. It is possible to understand that there is a straight relation between both Figures 4 and 5. Cracks appeared where isodisplacement lines tend to concentrate. At the beam's top, small cracks appear connecting reinforcement to concrete top surface. At the bottom, as reinforcement spacing is smaller, a single crack appears connecting all the bars and leading to the delamination of the concrete cover.

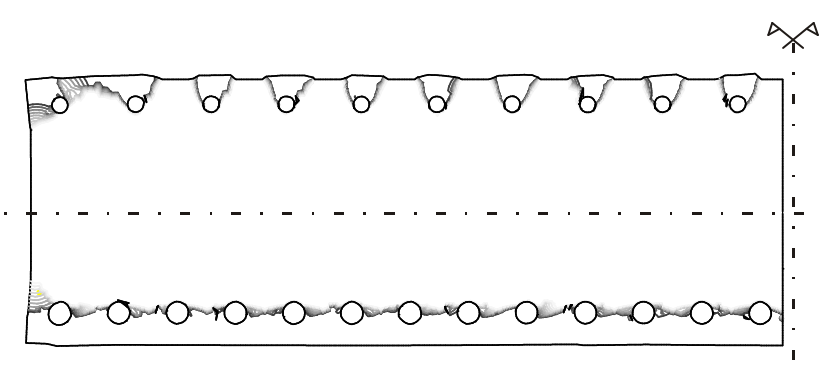

Figure 5. Isodisplacement lines showing cracks on concrete.

\section{$3.32 D$ longitudinal analysis}

For each corrosion level $X_{p}$, results obtained from the cross section analysis were used to build a 2D longitudinal model to check the load carrying capacity of the corroded structure.

In order to reach the compatibility between the cross section model and the 2D longitudinal model, it was necessary to project the damage variable from the first to the second model. Firstly, it was necessary to divide the cross section into horizontal slices. After this, the average damage on concrete for each slice was computed considering the parts of detached concrete with damage $d$ equal to 1 . Finally a horizontal projection of concrete average damage of each slice between both models was conducted and the 2D longitudinal model of the corroded structure built.
In the 2D longitudinal model, reinforced concrete was modeled as a composite material as proposed by Oliver et al. (2008) constituted by a matrix, representing concrete, with embedded fibers representing reinforcement. According to the mixture theory, a composite material is a continuum in which infinitesimal volume is occupied simultaneously by all constituents behaving as a parallel mechanical system. Consequently, all constituents are subjected to the same strain and stresses are given by the weighted sum, in terms of volume fraction, of the stresses of each constituent.

The ICDM, together with the CSDA and upgraded with the initial damage obtained from the cross section analysis, was used in order to model de deteriorated matrix behavior. For the fibers, and in order to account the bond-slip effect, the slippingfiber model proposed by Oliver et al. (2008) was adopted. This model mainly consists on the association of two components, representing reinforcement and its interface with concrete, in a serial system. The slipping fiber-strain $\varepsilon^{f}$ is given by the sum of the strains on both components:

$\varepsilon^{f}=\varepsilon^{d}+\varepsilon^{i}$

where $\varepsilon^{d}$ is the reinforcement mechanical deformation and $\varepsilon^{i}$ is the deformation due to sliding. For the serial system the stresses are identical in both components:

$\sigma^{f}=\sigma^{d}+\sigma^{i}$

Considering for each component an onedimensional elastoplastic model, the constitutive behavior of the serial system results also in an elastoplastic model with the following characteristics:

$$
\begin{aligned}
\sigma_{y}^{f} & =\min \left(\sigma_{y}^{d}, \sigma_{i}^{i}\right) \\
E^{f} & =\frac{1}{\frac{1}{E^{d}}+\frac{1}{E^{i}}}
\end{aligned}
$$

where $E^{d}$ and $\sigma_{y}{ }^{d}$ are the steel Young's modulus and yield stress, respectively, $E^{i}$ is the interface elastic modulus and $\sigma_{y}^{i}$ is the interface bond limit stress. When $E^{i} \rightarrow \infty$ and $\sigma_{y}^{d}<\sigma_{y}^{i}$, the system reproduces only the mechanical behavior of reinforcement considering perfect adhesion between concrete and steel bars. In this work, the hypothesis of having $E^{i} \rightarrow \infty$ and, for uncorroded state, $\sigma_{y}{ }^{d}=\sigma_{y}{ }^{i}$ was considered.

For the corroded states and in order to characterize bond strength deterioration, the M-pull model proposed by Bhargava et al. (2004) was adopted. This model, summarized in equation (19), gives the normalized bond strength $\sigma_{y}^{i}\left(X_{p}\right) / \sigma_{y}^{i}\left(X_{p}=0\right)$ as a function of the corrosion level $X_{p}$ and is based on the available experimental data. 
$\frac{\sigma_{y}^{i}\left(X_{p}\right)}{\sigma_{y}^{i}\left(X_{p}=0\right)}=\left\{\begin{array}{cl}1.0 & \text { if } X_{p} \leq 1.5 \% \\ 1.192 \cdot e^{-0.117 X_{p}} & \text { if } X_{p}>1.5 \%\end{array}\right.$

Summarizing, the present corrosion two step model is capable of taking into account the following effects: concrete deterioration and cracking due to corrosion products expansion; effective reinforcement area reduction and bond strength deterioration.

In Figure 6, the normalized force-displacement diagram is presented for several corrosion levels. The normalized force and displacement are given respectively by the ratio between the load carrying capacity and displacement of the corroded and uncorroded beam.

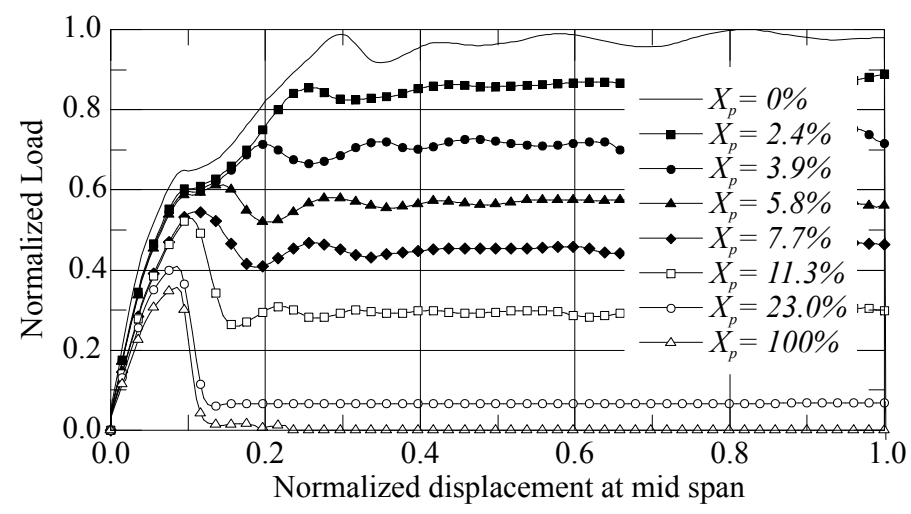

Figure 6. Normalized load/displacement diagram for several corrosion levels $X_{p}$.

Figure 6 confirms the expected flexural failure behavior for the beam due to the overdesign of the transversal reinforcement. Three behavior stages can be identified for the uncorroded state. The first stage corresponding to elasticity, the second equivalent to crack spreading and the third corresponding to mechanism development due to a plastic hinge formation at mid span. Regard that, for higher levels of corrosion, the second stage tend to disappear because when the first crack occurs the corroded reinforcement immediately enters into the plastic domain.

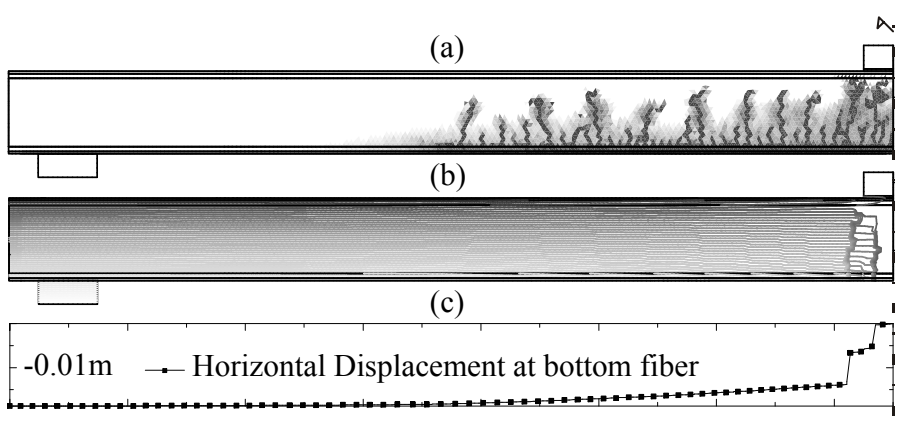

Figure 7. 2D longitudinal model results. (a) Damage variable $d$; (b) Isodisplacement lines showing the two main cracks corresponding to failure mechanism; (c) Horizontal displacement at bottom fiber showing the jump on the displacement field corresponding to the two main cracks.
In Figure 7(a), it is possible to observe the mechanism development captured with the 2D longitudinal model corresponding to the development of two large width cracks at mid span (see Figure 7(b)), which according to the CSDA kinematics, correspond to jumps on the displacement field (see Figure 7(c)).

\subsection{Robustness assessment}

As previously defined, and in order to assess robustness, the damage considered was the corrosion of the bottom longitudinal reinforcement and the structural performance was the load carrying capacity. In Figure 8 the normalized peak load carrying capacity is plotted as function of the corrosion level $X_{p}$. The normalized bond strength, given by the ratio between the corroded and uncorroded stresses is also plotted in Figure 8.

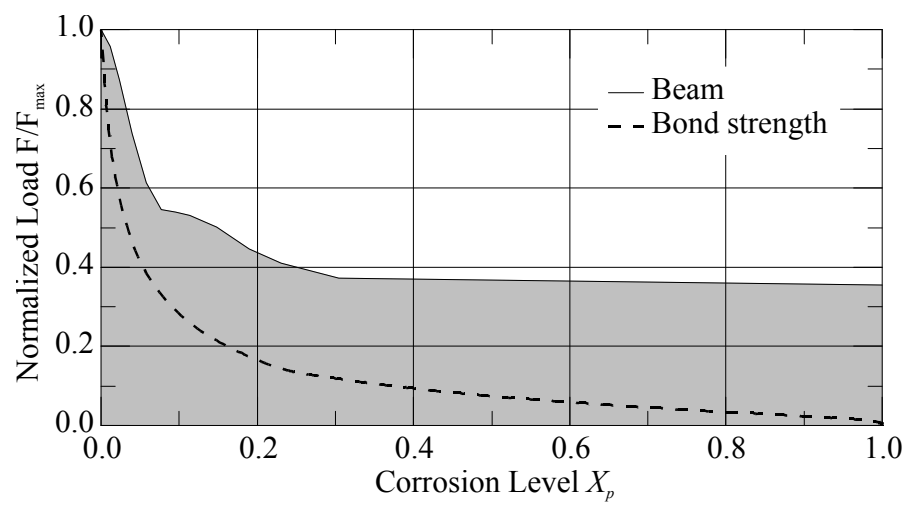

Figure 8 . Normalized peak load carrying capacity as a function of the corrosion level $X_{p}$.

From the analysis of Figure 8 , it is possible to conclude that for corrosion levels $X_{p}$ below $10 \%$, bond strength reduction is the major factor causing decreasing on peak load. For corrosion levels from $10 \%$ to $30 \%$, bond strength deterioration starts loosing influence and for higher corrosion levels structural load carrying capacity is provided only by the concrete material. The irregularities observed on the normalized load presented in Figure 8 are due to concrete cover spalling on both beam's bottom and top. The minimum residual load carrying capacity for the corroded structure is reached for corrosion levels higher than $30 \%$ and is about $36 \%$ of the load carrying capacity of the uncorroded beam.

Finally the robustness of the corroded beam can be assessed using equation (13) in order to calculate the area above the curve with respect to normalized load carrying capacity in Figure 8:

$R=\int_{0}^{1} F / F_{\max }\left(X_{p}\right) d X_{p}=0.42$

The robustness value of 0.42 gives an average rate of the carrying capacity loss subjected to a corrosion level that may vary from $0 \%$ to $100 \%$. This value contrasts with a robustness value of 1 for a simple concrete structure. Regard that, simple con- 
crete may seem less robust than the reinforced ones, but in fact they are not affected by reinforcement corrosion.

\section{CONCLUSIONS}

Robustness is an emergent concept related with the increased fear resulting from extreme events such as terrorist attacks. Corrosion of reinforced concrete structures is also an issue due to the expected costs on maintenance and replacements expected on the next decades.

In the present work, an effort to couple both referred issues was made. Therefore, several definitions and frameworks proposed by different authors were analyzed and discussed. From the proposed definitions it was concluded that some authors define robustness as a property of the structure and others define it also as a property of the structure environment.

Related to the proposed measures to assess robustness, it was concluded that they cannot be compared directly, because they measure different things.

Having in mind a structural property concept for robustness, a new definition was proposed. The corresponding deterministic measure was defined and illustrated with a practical example consisting on a simply supported beam subjected to corrosion of longitudinal reinforcement.

In order to assess robustness, an advanced methodology based on ICDM, CSDA, mixture theory and M-pull model for predicting bond strength deterioration was used.

Results show that the main cause of the drop on load carrying capacity of the studied beam is the bond strength deterioration. Concrete cracking due to corrosion products expansion and reinforcement area reduction has minor influence.

Robustness of the corroded beam resulted on 0.42 and the residual load carrying capacity of the full corroded specimen resulted on $36 \%$ of the uncorroded one.

\section{ACKNOWLEDGMENTS}

The authors would like to acknowledge COST Action TU-0601 and Fundação para Ciência e Tecnologia, scholarship SFRH/BD/45799/2008, for help supporting this research.

\section{REFERENCES}

Agarwal, J., England, J., and Blockley, D. (2006). Vulnerability analysis of structures. Structural Engineering International, 16(2): 124-128.
Baker, J., Schubert, M., and Faber, M. (2008). On the assessment of robustness. Structural Safety, 30(3): 253-267.

Bhargava, K., Ghosh, A., Mori,Y., and Ramanujam, S. (2007). Corrosion-induced bond strength degradation in reinforced concrete analytical and empirical models. Nuclear Engineering and Design, 237(11): 1140-1157.

Biondini, F. and Restelli, S. (2008). Damage propagation and structural robustness. In Life-Cycle Civil Engineering: Proceedings of the International Symposium on Life-Cycle Civil Engineering, IALCCE'08, Held in Varenna, Lake Como, Italy on June 11-14 2008, page 131. Taylor \& Francis.

Callaway, D., Newman, M., Strogatz, S., and Watts, D. (2000). Network robustness and fragility: Percolation on random graphs. Physical Review Letters, 85(25): 5468-5471.

Canisius, T., Sørensen, J., and Baker, J. (2007).Robustness of structural systems - a new focus for thejoint committee on structural safety (JCSS). In Proc., 10th Int. Conf. on Application of Statistic and Probability in Civil Engineering (ICASP10).

Cavaco, E. S. (2009). Robustness of Corroded Reinforced Concrete Structures. Technical report, Short Term Scientific Mission, Cost Action TU-0601, Barcelona.

Frangopol, D. M. and Curley, J. P. (1987). Effects of damage and redundancy on structural reliability. Journal of Structural Engineering, 113(7): 1533-1549.

Ghosn, M. and Moses, F. (1998). NCHRP Report 406: Redundancy in Highway Bridge Superstructures. Transportation Research Board, National Research Council, Washington, DC.

Lind, N. (1995). A measure of vulnerability and damage tolerance. Reliability engineering \& systems safety, 48(1):1-6.

NTSB (2008). Collapse of I-35W Highway Bridge. Highway Accident Report NTSB/HAR-08/03. Technical report, National Transportation Safety Board.

Oliver, J., Cervera, M., Oller, S., and Lubliner, J. (1990). Isotropic damage models and smeared crack analysis of concrete. In Second International Conference on Computer Aided Analysis and Design of Concrete Structures, volume 2, pages 945-958.

Oliver, J., Huespe, A., and Cante, J. (2007). An implicit/explicit integration scheme to increase computability of non-linear material and contact/friction problems. Computer Methods in Applied Mechanics and Engineering.

Oliver, J., Huespe, A., Pulido, M., and Chaves, E. (2002). From continuum mechanics to fracture mechanics: the strong discontinuity approach. Engineering Fracture Mechanics, 69(2): 113-136.

Oliver, J. ,Linero, D., Huespe, A., and Manzoli, O. (2008).Two-dimensional modeling of material failure in reinforced concrete by means of a continuum strong discontinuity approach. Computer Methods in Applied Mechanics and Engineering, 197(5): 332- 348.

Sánchez, P., Huespe, A., Oliver, J., and Toro, S. (2008). Numerical modelling of the load carrying capacity degradation in concrete beams due to reinforcement corrosion. In 8th World Congress on Computational Mechanics (WCCM8), 5th European Congress on Computational Methods in Applied Sciences and Engineering (ECCO $\neg M A S$ 2008).

Starossek, U., Haberland, M. (2008). Measures of structural robustness - requirements and applications. In Proceedings, ASCE SEI 2008 Structures Congress, Crossing Borders.

Starossek, U. (2009). Collapse types and robustness measures. In COST Action TU-0601 Robustness of Structures.

Wisniewski, D., Casas, J., and Ghosn, M. (2006). Load capacity evaluation of existing railway bridges based on robustness quantification. Structural Engineering International, $16(2): 161-166$. 